\title{
Short-time asymptotic expansions of semilinear evolution equations
}

\author{
Matthias A. Fahrenwaldt \\ Institut für Mathematische Stochastik, Leibniz Universität Hannover, \\ Welfengarten 1, 30167 Hannover, Germany \\ (fahrenw@stochastik.uni-hannover.de)
}

(MS received 4 August 2014; accepted 14 November 2014)

\begin{abstract}
We develop an algebraic approach to constructing short-time asymptotic expansions of solutions of a class of abstract semilinear evolution equations. The expansions are typically valid for both the solution of the equation and its gradient. We apply a perturbation approach based on the symbolic calculus of pseudo-differential operators and heat kernel methods. The construction is explicit and can be done to arbitrary order. All results are rigorously formulated in terms of Banach algebras. As an application we obtain a novel approach to finding approximate solutions of Markovian backward stochastic differential equations.

Keywords: semilinear equations; asymptotic analysis; Banach algebras; backward stochastic differential equations

2010 Mathematics subject classification: Primary 35K58
\end{abstract}

Secondary 35B40

\section{Introduction}

Asymptotic expansions are a well-established tool in the qualitative analysis of partial differential equations (PDEs) in general and (semilinear) evolution equations in particular. Asymptotics are typically used to provide approximate solutions or to examine the short- or long-time behaviour of solutions. However, an asymptotic expansion of the solution may not simultaneously yield information about its gradient, which can be a significant drawback of this technique.

We consider abstract semilinear evolution equations of the form

$$
\begin{aligned}
\partial_{t} u(t) & =A u(t)+F\left(t, u(t), T_{1} u(t), \ldots, T_{n} u(t)\right), \\
u(0) & =f,
\end{aligned}
$$

in a suitable Banach algebra $X$. The algebraic structure is required to make sense of the nonlinearity expressed by the forcing term $F$. Here, the $T_{i}$ are linear operators essentially representing differentiation. Our aim is to perturbatively construct asymptotic expansions in $X$ as $t \rightarrow 0^{+}$of the form $u(t) \sim u_{0}(t)+u_{1}(t) t+u_{2}(t) t^{2}+\cdots$ such that also $T_{i} u(t) \sim T_{i} u_{0}(t)+T_{i} u_{1}(t) t+T_{i} u_{2}(t) t^{2}+\cdots$ for $i=1, \ldots, n$.

To this end we suppose that $A$ generates an analytic semigroup $G(t)$ on $X$ that may be expanded $G(t) \sim G_{0}(t)+G_{1}(t) t+G_{2}(t) t^{2}+\cdots$ for families of bounded 
linear operators $G_{j}$, usually induced by a heat kernel expansion. Moreover, we assume that $F$ is analytic in its arguments. Using holomorphic functional calculus in several variables, we then recursively construct an asymptotic expansion of the PDE solution. The functions $u_{i}$ are given recursively as

$$
\begin{aligned}
& u_{0}(t)=G_{0}(t) f \\
& u_{i}(t)=G_{i}(t) f+\frac{1}{t^{i}} \int_{0}^{t} \varphi_{i-1}\left(t, s ; u_{0}, \ldots, u_{i-1}\right) \mathrm{d} s \quad \text { for } i \geqslant 1 .
\end{aligned}
$$

Here,

$$
\varphi_{i}\left(t, s ; u_{0}, \ldots, u_{i}\right)=\sum_{j=0}^{i}(t-s)^{j} s^{i-j} G_{j}(t-s) F_{i-j}\left(s ; u_{0}, \ldots, u_{i}\right),
$$

and the $F_{k}$ are functions $[0, T] \rightarrow X$ that are polynomial in $u_{l}, T_{j} u_{l}(l=0,1, \ldots, k$ and $j=1, \ldots, n)$ with coefficients given in terms of derivatives of $F$; they can be constructed explicitly.

Typical spaces to which our perturbation ansatz applies are uniform function spaces and Sobolev spaces on certain bounded domains.

The reason for making the analyticity assumption is as follows. One could naively apply the multivariate Taylor theorem to $F\left(t, u(t), T_{1} u(t), \ldots, T_{n} u(t)\right)$ by exploiting the multiplicative structure of $X$. However, this does not necessarily yield an asymptotic expansion of both $u$ and $T_{i} u$ that is valid in $X$ as $t \rightarrow 0^{+}$, since the error term in Taylor's theorem may not be controllable in terms of the norm on $X$.

Qualitative approximations of PDE solutions via asymptotics are studied from both pure and applied mathematics perspectives. On the pure side we want to understand the 'mechanics' of the solution and see how it depends on the data: the initial condition, the forcing term and the geometry of the underlying manifold. Many problems in applied mathematics, e.g. financial mathematics, are formulated as backward stochastic differential equations (BSDEs), which can be recast as semilinear PDEs in the Markovian case. Short-time approximate solutions are important, for example, in the pricing of options near expiry, as they allow one to infer data (e.g. volatility) from observed market prices; the gradient of the solution is economically crucial, as it expresses the hedging strategy.

Short-time asymptotic expansions for linear PDEs can be constructed by heat kernel methods. This is widely applied in pure (e.g. differential geometry [4]) and applied branches such as theoretical physics [30] and financial mathematics $[12,16]$. We do not know of a translation of this approach to nonlinear PDEs.

We illustrate the framework in two examples that highlight the simultaneous approximation of the function and its gradient:

(i) a parabolic semilinear evolution equation with a quadratic forcing term on a bounded domain; and

(ii) explicit short-time asymptotics for stochastic processes that are BSDE solutions, derived by exploiting the link with Markovian BSDEs.

This paper is organized as follows. Section 2 introduces the notation for function spaces and summarizes preliminaries on Banach algebras and topological tensor 
products. Section 3 describes the set-up, states and motivates the assumptions and gives the key results. These are proved in $\S 4$. In $\S 5$ we illustrate the framework with examples.

\section{Preliminaries}

To make the paper largely self-contained we here summarize some background material.

\subsection{Function spaces (see [1])}

Let $\Omega \subseteq \mathbb{R}^{n}$ be an open domain in $\mathbb{R}^{n}$, let $\partial \Omega$ be its boundary and let $\bar{\Omega}$ be its closure in the standard topology of $\mathbb{R}^{n}$. We denote by $C(\Omega)$ the linear space of bounded continuous functions $\Omega \rightarrow \mathbb{R}$. This is a metric space under the usual supremum norm $\|f\|_{\infty}=\sup _{x \in \Omega}|f(x)|$.

For a given Banach space $X$ with norm $\|\cdot\|_{X}$, we denote the set of continuous functions $[0, T] \rightarrow X$ by $C([0, T] ; X)$. If $X=\mathbb{R}$, we simply write $C([0, T])$. This space can also be turned into a Banach space under the norm $\|f\|=\sup _{t \in[0, T]}\|f(t)\|_{X}$. Note that $C([0, T] ; C(\Omega)) \subseteq C([0, T] \times \Omega)$ with strict inclusion for $\Omega$ unbounded.

We call an $n$-tuple of non-negative integers $\alpha=\left(\alpha_{1}, \ldots, \alpha_{n}\right)$ a multi-index. For $D_{j}=\partial_{j}=\partial / \partial x_{j}$ and a multi-index $\alpha$ we define $D^{\alpha}=D_{1}^{\alpha_{1}} \cdots D_{n}^{\alpha_{n}}$ to be a differential operator of order $|\alpha|=\alpha_{1}+\cdots+\alpha_{n}$. We denote the gradient operator by $D=\left(D_{1}, \ldots, D_{n}\right)$.

For $k$ a non-negative integer we let $C^{k}(\Omega)$ be the space of complex-valued $k$-times continuously differentiable functions with norm

$$
\|f\|_{C^{k}(\Omega)}=\sum_{|\alpha| \leqslant k} \frac{1}{|\alpha| !}\left\|D^{\alpha} f\right\|_{\infty} .
$$

This norm turns $C^{k}(\Omega)$ into an algebra under pointwise operations; $C^{k}(\bar{\Omega})$ becomes a Banach algebra. Similarly, we denote by $C^{1,2}([0, T] \times \Omega)$ the space of functions $[0, T] \times \Omega \rightarrow \mathbb{R}$ that are once continuously differentiable with respect to $t \in[0, T]$ and twice continuously differentiable with respect to $x \in \Omega$.

For open $U \subset \mathbb{C}^{n}$ we denote by $O(U)$ the algebra of holomorphic functions on $U$. If $U=\bigcup_{i=1}^{\infty} K_{i}$ with $K_{i}$ compact, then $O(U)$ becomes a Fréchet algebra under the semi-norms $p_{j}(f)=\sup _{z \in K_{j}}|f(z)|$.

The Lebesgue spaces $L^{p}(\Omega)$ are defined as the equivalence classes of measurable real- or complex-valued functions $f$ on $\Omega$ such that

$$
\|f\|_{p}=\int_{\Omega}|f(x)|^{p} \mathrm{~d} x
$$

is finite.

\subsection{Banach algebras (see [2])}

We denote the set of linear operators $T: X \rightarrow Y$ between Banach spaces $X$ and $Y$ by $\mathcal{B}(X, Y)$, or simply by $\mathcal{B}(X)$ if $T$ is an endomorphism of $X$. The norm of such an operator is denoted by $\|T\|_{\mathcal{B}(X, Y)}$, or simply by $\|T\|$ if there is no danger of 
confusion. The complexification of a Banach algebra $X$ is defined by $X_{\mathbb{C}}=X \otimes_{\mathbb{R}} \mathbb{C}$. The algebra $X_{\mathbb{C}}$ is a Banach algebra under the usual multiplication

$$
\left(a_{1}+\mathrm{i} b_{1}\right)\left(a_{2}+\mathrm{i} b_{2}\right)=\left(a_{1} a_{2}-b_{1} b_{2}\right)+\mathrm{i}\left(a_{1} b_{2}+a_{2} b_{1}\right)
$$

and norm

$$
\|a+\mathrm{i} b\|_{X_{\mathbb{C}}}^{2}=\|a\|_{X}^{2}+\|b\|_{X}^{2} .
$$

If $X$ is a unital Banach algebra, the so is $X_{\mathbb{C}}$ with unit $1=1_{X_{\mathbb{C}}}=1_{X}+$ i0. Denote the spectrum of an element $a \in X_{\mathbb{C}}$ by $\operatorname{Sp}(a)=\left\{a \in X_{\mathbb{C}}: a\right.$ is not invertible in $\left.X_{\mathbb{C}}\right\}$. The resolvent set $\rho(a)$ is the complement of $\operatorname{Sp}(a)$ in $\mathbb{C}$.

\subsection{Analytic semigroups (see [20])}

For an unbounded operator $A$ on a Banach space $X$, let $D(A) \subset X$ be the domain of $A$. An operator $A: D(A) \rightarrow X$ is called sectorial if there are constants $\omega \in \mathbb{R}$, $\theta \in\left(\frac{1}{2} \pi, \pi\right), M>0$ such that

(i) the angular domain $S_{\theta, \omega}=\{\lambda \in \mathbb{C}: \lambda \neq \omega,|\arg (\lambda-\omega)|<\theta\}$ is a subset of the resolvent set $\rho(A)$ and

(ii) the operator norm of the resolvent of $A$ obeys the bound $\left\|(\lambda 1-A)^{-1}\right\|_{\mathcal{B}(X)} \leqslant$ $M /|\lambda-\omega|$ for $\lambda \in S_{\theta, \omega}$.

A sectorial operator yields an analytic semigroup $G(t)$ on $X$ by the Dunford integral

$$
G(t)=\frac{1}{2 \pi \mathrm{i}} \int_{\omega+\gamma_{r, \eta}} \mathrm{e}^{t \lambda}(\lambda 1-A)^{-1} \mathrm{~d} \lambda,
$$

where $r>0, \eta \in\left(\frac{1}{2} \pi, \theta\right)$ and $\gamma_{r, \eta}$ is the curve

$$
\{\lambda \in \mathbb{C}:|\arg \lambda|=\eta,|\lambda| \geqslant r\} \cup\{\lambda \in \mathbb{C}:|\arg \lambda| \leqslant \eta,|\lambda|=r\}
$$

oriented counter-clockwise. The operator $A$ is the infinitesimal generator of the semigroup $G(t)$. To a semigroup $G(t)$ with infinitesimal generator $A$ we associate intermediate spaces $D_{A}(\alpha, p)=\left\{x \in X: t \mapsto\left\|t^{1-\alpha-1 / p} A G(t) x\right\| \in L^{p}(0,1)\right\}$, where $\alpha \in(0,1)$ and $1 \leqslant p \leqslant \infty$.

\subsection{Topological tensor products (see [14])}

For $E, F$ vector spaces over $\mathbb{C}$ we denote the algebraic tensor product by $E \otimes F$. It is a vector space spanned by finite linear combinations $\sum_{i=1}^{N} e_{i} \otimes f_{i}$ for $\lambda_{i} \in \mathbb{C}, e_{i} \in$ $E, f_{i} \in F$. If $E$ and $F$ are Fréchet spaces with semi-norms $p_{i}$ and $q_{j}$, respectively, then there are semi-norms $r_{i j}$ on $E \otimes F$ defined as

$$
r_{i j}(g)=\inf \left\{\sum_{k=1}^{N} p_{i}\left(e_{k}\right) q_{j}\left(f_{k}\right) \mid g=\sum_{k=1}^{N} e_{k} \otimes f_{k}\right\} .
$$

The completion of $E \otimes F$ under the topology induced by these semi-norms is the projective tensor product denoted by $E \hat{\otimes}_{\pi} F$. Any $g \in E \hat{\otimes}_{\pi} F$ can be (non-uniquely) written as

$$
g=\sum_{k=1}^{\infty} \lambda_{k} e_{k} \otimes f_{k}
$$


with $\sum_{k=1}^{\infty}\left|\lambda_{k}\right| \leqslant 1$ and $e_{k}, f_{k} \rightarrow 0$ as $k \rightarrow \infty$ in $E$ and $F$, respectively (see [14, theorem 1, ch. 1, $\S 1]$ ).

The projective tensor product has the following universal property (the same result can be extended to the tensor product of finitely many spaces).

Proposition 2.1 (see [14, proposition 1, ch. 1]). Let E, F be Fréchet spaces and let $E \hat{\otimes}_{\pi} F$ be their projective tensor product. Then for every Fréchet space $G$ and every bilinear map $\Phi: E \times F \rightarrow G$ there is a unique linear map $\bar{\Phi}: E \hat{\otimes}_{\pi} F \rightarrow G$ such that the following diagram commutes:

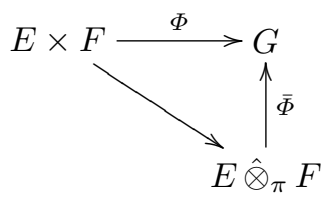

where the map $E \times F \rightarrow E \hat{\otimes}_{\pi} F$ is the canonical inclusion.

\section{Statement of the key result}

We motivate and state the key results of this paper. Our notion of an asymptotic expansion is as follows.

Definition 3.1. Let $T>0$ and let $f$ be a function on $[0, T]$ with values in a Banach space $X$. A formal series $\sum_{k=0}^{\infty} a_{k}(t) t^{k}$, where the $a_{k}$ are bounded functions $[0, T] \rightarrow X$ is called an asymptotic expansion for $f$ in $X$ near $t=0$ if, for each positive integer $m$, there is a constant $c_{m}$ with

$$
\left\|f(t)-\sum_{k=0}^{m} a_{k}(t) t^{k}\right\|_{E} \leqslant c_{m} t^{m+1}
$$

for all sufficiently small $t$. We then write $f(t) \sim \sum_{k=0}^{\infty} a_{k}(t) t^{k}$ in $X$ as $t \rightarrow 0^{+}$.

Now let $X$ be a Banach space. We consider a semilinear autonomous evolution equation in $X$ of the form

$$
\left.\begin{array}{rl}
\partial_{t} u(t) & =A u(t)+F\left(t, u(t), T_{1} u(t), \ldots, T_{n} u(t)\right), \\
u(0) & =f
\end{array}\right\}
$$

where $A: D(A) \subseteq X \rightarrow X$ is a linear operator, $F:[0, T] \times X^{n+1} \rightarrow X$ and $T_{j}: D\left(T_{j}\right) \subseteq X \rightarrow X$ are linear. More detailed assumptions follow.

REMARK 3.2. All assumptions and results carry over to the non-autonomous case when $A$ depends on time $t$ by replacing semigroups with evolution systems.

If the operator $A$ generates an analytic semigroup $G(t)$ on $X$, we can convert the PDE (3.1) into the integral equation

$$
u(t)=G(t) f+\int_{0}^{t} G(t-s) F\left(s, u, T_{1} u, \ldots, T_{n} u\right) \mathrm{d} s
$$


by Duhamel's principle (variation of constants). A solution of this equation is called a mild solution of the evolution equation (3.1).

To fix ideas and to motivate assumptions, consider the following simple example.

EXAMPLE 3.3. Let $\Omega$ be a bounded domain in $\mathbb{R}^{n}$ with smooth boundary $\partial \Omega$, and consider the semilinear Dirichlet problem

$$
\left.\begin{array}{c}
\partial_{t} u=\Delta u+F\left(t, u, D_{1} u, \ldots, D_{n} u\right) \quad \text { on }(0, T] \times \Omega, \\
u=0 \quad \text { on }(0, T] \times \partial \Omega, \\
u=f \quad \text { on }\{0\} \times \Omega,
\end{array}\right\}
$$

for some $T>0$ with $\Delta$ the Laplace operator $\Delta=\sum_{j=1}^{n} D_{j}^{2}$. Clearly, $\Delta$ is sectorial and generates an analytic semigroup $G(t)$ in the Banach space $X=C(\bar{\Omega})$ (see $[20$, ch. 3.1.5]). Let $U \subset \mathbb{R}$ be an open set such that the range of $\left(f, D_{1} f, \ldots, D_{n} f\right)$ is contained in $U^{n+1}$. The function $F:[0, T] \times \bar{\Omega} \times U \times U^{n}$ is assumed to be smooth in its arguments. By $[29$, proposition 3.1], the PDE has a unique (mild) solution in $C\left(\left[0, T_{0}\right] ; C^{1}(\bar{\Omega})\right)$ for some $T_{0}>0$.

The aim is now to derive the asymptotics of $u$ on small time intervals by taking a rigorous perturbation approach: formally, assume that $u \sim \sum_{i=0}^{\infty} u_{i}(t) t^{i}$ and also that $G(t) \sim \sum_{i=0}^{\infty} G_{i}(t) t^{i}$. Then expand the left- and right-hand sides of (3.2) and match the terms according to their order in $t$. The key problem is to expand $F\left(t, u, T_{1} u, \ldots, T_{n} u\right)$, which requires a multiplicative structure in the space $X$.

To make this paper self-contained, we begin with assumptions that guarantee the existence of an analytic semigroup with infinitesimal generator $A$ and the existence of a mild solution of the PDE (3.1).

HYPOTHESIS 3.4 (existence of a short-time solution). Assume the following.

(i) Semigroup generation: $X$ is a Banach space and $A: D(A) \rightarrow X$ is a linear sectorial operator.

(ii) Intermediate space: there exist an $\alpha \in(0,1)$ and a Banach space $X_{\alpha}$ such that

(a) the injection $\imath: X_{\alpha} \hookrightarrow X$ is continuous,

(b) $X_{\alpha}$ is an intermediate space, i.e. $D_{A}(\alpha, 1) \subseteq X_{\alpha} \subseteq D_{A}(\alpha, \infty)$,

(c) the restriction of $A$ to $X_{\alpha}$ is sectorial.

(iii) Forcing term F:

(a) the map $F:[0, T] \times X^{n+1} \rightarrow X$ is a continuous function such that for every $R>0$ there is a constant $L$ with

$$
\left\|F\left(t, x, T_{1} x, \ldots, T_{n} x\right)-F\left(t, y, T_{1} y, \ldots, T_{n} y\right)\right\|_{X} \leqslant L\|x-y\|_{X}
$$

for $\|x\|_{X},\|y\|_{X} \leqslant R$

(b) the linear operators $T_{i}: X_{\alpha} \rightarrow X$ are bounded.

In example 3.3, we have $X=C(\bar{\Omega}), \alpha=\frac{1}{2}, X_{\alpha}=C_{\mathcal{B}}^{1}(\bar{\Omega})=\left\{u \in C^{1}(\bar{\Omega}): u=\right.$ 0 on $\partial \Omega\}$ and the $T_{j}$ are given by the differential operators $D_{j}$.

The assumptions give us a unique mild solution of (3.1). 
Proposition 3.5 (Lunardi [20, theorems 7.1.2 and 7.1.3]). Let $\alpha \in(0,1)$ and let $\overline{D(A)}^{\alpha}$ be the closure of the domain of $A$ in the space $X_{\alpha}$. Then, under hypothesis 3.4, for every $f \in \overline{D(A)}^{\alpha}$, the evolution equation (3.1) has a unique mild solution $u \in C\left(\left[0, T_{0}\right] ; X\right) \cap C\left(\left[0, T_{0}\right] ; X_{\alpha}\right)$ for some $T_{0}>0$.

We now impose additional hypotheses in order to construct asymptotic expansions.

Hypothesis 3.6 (existence of short-time asymptotics). Let $u$ be the unique mild solution of (3.1).

(i) Algebra: the space $X$ is a unital commutative Banach algebra.

(ii) Heat kernel expansion: there is an asymptotic expansion of $G(t)$ of the form

$$
G(t) \sim G_{0}(t)+G_{1}(t) t+G_{2}(t) t^{2}+\cdots
$$

in $\mathcal{B}\left(X, X_{\alpha}\right)$ as $t \rightarrow 0^{+}$.

(iii) Functional calculus: the function $F$ belongs to $C([0, T]) \hat{\otimes}_{\pi} X \hat{\otimes}_{\pi} O(U)$, where $U \subset \mathbb{C}^{n+1}$ is an open neighbourhood of both $\operatorname{Sp}(u) \times \operatorname{Sp}\left(T_{1} u\right) \times \cdots \times \operatorname{Sp}\left(T_{n} u\right)$ and $\operatorname{Sp}\left(u_{0}\right) \times \operatorname{Sp}\left(T_{1} u_{0}\right) \times \cdots \times \operatorname{Sp}\left(T_{n} u_{0}\right)$ with $u$ the solution of (3.1) and $u_{0}=G_{0} f$ (all spectra in $X \otimes_{\mathbb{R}} \mathbb{C}$ ).

A word of motivation and comment on the hypotheses.

- Algebra: the Banach algebra $X$ is typically realized as a uniform space. However, the framework also covers spaces of Hölder continuous functions and Sobolev spaces on bounded domains that satisfy the cone condition (see $[1$, ch. 4]).

- Heat kernel expansion: the operators $G, G_{j}$ are smoothing $X \rightarrow X_{\alpha}$ so as to yield a mild solution in $X_{\alpha}$.

The existence of a heat kernel expansion typically hinges on $A$ being uniformly elliptic and having smooth coefficients. Non-uniformly elliptic operators with heat kernel expansions include the Laplacian on hyperbolic space, sub-elliptic operators and Kolmogorov operators (see [6]).

The terms $G_{j}$ can be obtained for heat kernels on compact manifolds with or without boundary in various ways, e.g. by the Levi parametrix method $[4,21]$ or pseudo-differential operator techniques $[13,28]$.

We obtain a simple perturbation approach by setting $G_{0}(t)=G(t)$ and $G_{j}(t)=0$ for $j \geqslant 1$.

- Functional calculus: our first impulse would be to use a formal Taylor series for $F$. However, this will not yield an asymptotic expansion of $u$ in $X$, as the remainder term in Taylor's theorem gives estimates in the supremum norm that may not be controllable by the norm in $X$ (consider the case of Sobolev spaces). Hence, we require that $F$ be analytic so as to use holomorphic functional calculus. 
The space in which $F$ lives looks unusual. However, just revert to example 3.3: without loss of generality, $F \in C\left([0, T] \times \bar{\Omega} \times \bar{U} \times \bar{U}^{n}\right)$, which is isomorphic to $C([0, T]) \hat{\otimes}_{\pi} C(\bar{\Omega}) \hat{\otimes}_{\pi} C\left(\bar{U}^{n+1}\right)$. Thus, condition (iii) of hypothesis 3.6 is the natural generalization of the example to the situation that allows holomorphic functional calculus.

Loosely speaking (this is made precise in $\S 4.3$ ), the action of $F$ on $X_{\alpha}$ and $X$ is constructed as follows: any $F \in C([0, T]) \hat{\otimes}_{\pi} X \hat{\otimes}_{\pi} O(U)$ can be represented as

$$
F=\sum_{j=0}^{\infty} \lambda_{j} f_{j} \otimes y_{j} \otimes g_{j}
$$

with $\lambda_{j} \in \mathbb{C}, f_{j} \in C([0, T]), y_{j} \in X$ and $g_{j} \in O(U)$. We thus, by abuse of notation, set $F\left(t, x, T_{1} x, \ldots, T_{n} x\right)=\sum_{j=0}^{\infty} \lambda_{j} f_{j}(t) y_{j} g_{j}\left(x, T_{1} x, \ldots, T_{n} x\right)$, where the maps $g_{j}\left(x, T_{1} x, \ldots, T_{n} x\right)$ are defined by functional calculus on $X$.

The main result of this paper now reads as follows.

Theorem 3.7. Let $u \in C\left([0, T] ; X_{\alpha}\right)$ be a mild solution of (3.1). Then, under hypotheses 3.4 and 3.6, the following hold.

(i) There are functions $u_{i} \in C\left(\left[0, T_{0}\right] ; X_{\alpha}\right)$ such that

$$
u(t) \sim \sum_{i=0}^{\infty} u_{i}(t) t^{i}
$$

in $X_{\alpha}$ as $t \rightarrow 0^{+}$. The functions $u_{i}$ are given recursively as

$$
\begin{aligned}
& u_{0}(t)=G_{0}(t) f \\
& u_{i}(t)=G_{i}(t) f+\frac{1}{t^{i}} \int_{0}^{t} \varphi_{i-1}\left(t, s ; u_{0}, \ldots, u_{i-1}\right) \mathrm{d} s \quad \text { for } i \geqslant 1 .
\end{aligned}
$$

Here,

$$
\varphi_{i}\left(t, s ; u_{0}, \ldots, u_{i}\right)=\sum_{j=0}^{i}(t-s)^{j} s^{i-j} G_{j}(t-s) F_{i-j}\left(s ; u_{0}, \ldots, u_{i}\right),
$$

and the $F_{k}$ are functions $[0, T] \rightarrow X$ that are polynomial in $u_{l}, T_{j} u_{l}(l=$ $0,1, \ldots, k$ and $j=1, \ldots, n)$ with coefficients given in terms of derivatives of $F$. The $F_{k}$ can be explicitly constructed with lowest order terms given by

$$
\begin{aligned}
& u_{0}(t)=G_{0}(t) f \\
& u_{1}(t)=G_{1}(t) f+\frac{1}{t} \int_{0}^{t} G_{0}(s) F\left(u_{0}(s), T_{1} u_{0}(s), \ldots, T_{n} u_{0}(s)\right) \mathrm{d} s .
\end{aligned}
$$

(ii) The images of $u$ under the $T_{j}(j=1, \ldots, n)$ have the asymptotic expansion

$$
T_{j} u(t) \sim \sum_{i=0}^{\infty}\left(T_{j} u_{i}\right)(t) t^{i}
$$

which is valid in $X$ as $t \rightarrow 0^{+}$. 
Note that each term $u_{i}$ is of the same structure as (3.2) in that it is given as an action on the initial datum plus a time integral over a forcing term. If $G_{i}(t)$ is an analytic semigroup, then $u_{i}$ satisfies a PDE obtained by converting the integral equation for $u_{i}$ into a PDE.

An important special case of the above expansion is given by setting $G_{0}=G$ and $G_{i}=0$ for $i \geqslant 1$. The expansion then becomes $u_{0}=G f$ and

$$
u_{i}(t)=\frac{1}{t^{i}} \int_{0}^{t} s^{i-1} G(s) F_{i-1}\left(s ; u_{0}, \ldots, u_{i-1}\right) \mathrm{d} s .
$$

This leads to a coarser asymptotic expansion in the sense that each $u_{i}$ is composed of fewer terms than in the general case. Also, $u_{0}$ is a mild solution of the linear PDE

$$
\begin{gathered}
\partial_{t} v=A v, \\
v(0)=f,
\end{gathered}
$$

and for every $i \geqslant 1$ the function $t^{i} u_{i}(t)$ is the mild solution of the PDE

$$
\left.\begin{array}{rl}
\partial_{t} v & =A v+F_{i-1}\left(t ; u_{0}, \ldots, u_{i-1}\right), \\
v(0) & =0,
\end{array}\right\}
$$

which follows from (3.4) by standard semigroup methods.

The $F_{k}$ can be formally obtained by expanding $F\left(t, x, z_{0}, z_{1}, \ldots, z_{n}\right)$ as a Taylor series in the variables $z_{0}, \ldots, z_{n}$, replacing $z_{0}$ by $\sum u_{i}(t) t^{i}$ and the $z_{j}$ by the formal series $\sum T_{j} u_{i}(t) t^{i}$ for $j \geqslant 1$ and then collecting terms according to the order in $t$ after multiplying out.

\section{Proof of the key result}

The heart of the proof is to define $F\left(t, T_{1} u(t), \ldots, T_{n} u(t)\right)$ by holomorphic functional calculus via a Cauchy-type formula and then to use methods from the symbolic calculus of pseudo-differential operators [19].

As the holomorphic functional calculus requires the underlying Banach algebra to be over $\mathbb{C}$, we work in $X_{\mathbb{C}}$ from now on. By abuse of notation, we write $X$ but always mean the complexified algebra. We denote by 1 the unit of $X$.

\subsection{Asymptotic calculus}

We first develop a basic calculus of asymptotic expansions in the sense of definition 3.1, comprising products, formal inverses and the action of bounded operators.

LEMMA 4.1 (product expansions). Let $x_{1}, \ldots, x_{n}$ be functions $[0, T] \rightarrow X$ such that there are functions $x_{i, k_{i}}$ with

$$
x_{i}(t) \sim \sum_{k_{i}=0}^{\infty} x_{i, k_{i}}(t) t^{k_{i}}
$$

in $X$ as $t \rightarrow 0^{+}$. Then there are functions $b_{k}:[0, T] \rightarrow X$ given by

$$
b_{k}(t)=\sum_{k_{1}+\cdots+k_{n}=k} x_{1, k_{1}}(t) x_{2, k_{2}}(t) \cdots x_{n, k_{n}}(t)
$$


such that

$$
x_{1}(t) x_{2}(t) \cdots x_{n}(t) \sim \sum_{k=0}^{\infty} b_{k}(t) t^{k}
$$

in $X$ as $t \rightarrow 0^{+}$.

Proof. Given $m \in N$, we have by assumption that, for every $N \geqslant m$,

$$
\left\|x_{i}(t)-\sum_{k_{i}=0}^{N} x_{i, k_{i}}(t) t^{k_{i}}\right\|_{X} \leqslant c_{m}^{i} t^{m+1}
$$

for some constants $c_{m}^{(i)}$. Then (upon omitting the $t$-dependence) we have

$$
\begin{aligned}
x_{1} \cdots x_{n}-\sum_{k=0}^{N} b_{k} t^{k} & =\prod_{i=1}^{n}\left[x_{i}-\sum_{k_{i}=0}^{N} x_{i, k_{i}} t^{k_{i}}+\sum_{k_{i}=0}^{N} x_{i, k_{i}} t^{k_{i}}\right]-\sum_{k=0}^{N} b_{k} t^{k} \\
& =R+\prod_{i=1}^{n}\left[\sum_{k_{i}=0}^{N} x_{i, k_{i}} t^{k_{i}}\right]-\sum_{k=0}^{N} b_{k} t^{k} .
\end{aligned}
$$

Here, $R$ is a sum of products, where each product contains at least one factor of the form $x_{j}-\sum_{k_{j}=0}^{N} x_{j, k_{j}} t^{k_{j}}$. By (4.1), the norm of $R$ is bounded by $c_{m, 1} t^{m+1}$ for some $C_{m, 1} \in \mathbb{R}$. By construction of the $b_{k}$, the difference in (4.2) is also bounded in the norm by $c_{m, 2} t^{m+1}$ for some $c_{m, 2} \in \mathbb{R}$. Hence,

$$
\left\|x_{1}(t) \cdots x_{n}(t)-\sum_{k=0}^{N} b_{k}(t) t^{k}\right\|_{X} \leqslant c_{m} t^{m+1}
$$

for a constant $c_{m}$.

Lemma 4.2 (inverses). Suppose $u(t) \sim \sum_{i=0}^{\infty} u_{i}(t) t^{i}$ in $X$ as $t \rightarrow 0^{+}$and choose $\lambda \in$ $\mathbb{C}$ such that $\lambda \notin \mathrm{Sp}\left(u_{0}(t)\right)$ for any $t \in[0, T]$. Then there are universal polynomials $p_{i, j}$ such that setting

$$
v_{i}(t)=\sum_{j=1}^{i} p_{i, j}\left(u_{1}(t), \ldots, u_{i}(t)\right)\left(\lambda-u_{0}(t)\right)^{-(j+1)}
$$

leads to $(\lambda-u(t))^{-1} \sim \sum_{i=0}^{\infty} v_{i}(t) t^{i}$ in $X$ as $t \rightarrow 0^{+}$.

Proof. Start with the relation between formal power series,

$$
\left(\lambda-\sum_{i=0}^{\infty} u_{i}(t) t^{i}\right) \sum_{i=0}^{\infty} v_{i}(t) t^{i}=1,
$$

to derive formulae for the $v_{j}$. By considering $i=0$, we have that

$$
v_{0}=\left(\lambda-u_{0}(t)\right)^{-1}
$$

in the lowest order. Moreover, requiring that all other terms in the formal series (4.4) vanish, we have for $i \geqslant 1$ that

$$
\left(\lambda-u_{0}\right) v_{i}-\sum_{j=1}^{i} u_{j} v_{i-j}=0,
$$


or

$$
v_{i}=\left(\lambda-u_{0}\right)^{-1} \sum_{j=1}^{i} u_{j} v_{i-j} .
$$

The result follows by induction.

To check that this really yields an asymptotic expansion of $(\lambda-u)^{-1}$ we let $n \in \mathbb{N}, m \geqslant n$ and consider (all norms in $X$ )

$$
\begin{aligned}
& \left\|(\lambda-u)^{-1}-\sum_{j=0}^{n} v_{j}(t) t^{j}\right\| \\
& =\left\|(\lambda-u)^{-1}\left(1-(\lambda-u) \sum_{j=0}^{n} v_{j}(t) t^{j}\right)\right\| \\
& \leqslant\left\|(\lambda-u)^{-1}\right\| \\
& \quad \times\left\|1-\left[(\lambda-u)-\left(\lambda-\sum_{i=0}^{m} u_{i}(t) t^{i}\right)+\left(\lambda-\sum_{i=0}^{m} u_{i}(t) t^{i}\right)\right] \sum_{j=0}^{n} v_{j}(t) t^{j}\right\| \\
& \leqslant\left\|(\lambda-u)^{-1}\right\|\left(S_{1}+S_{2}\right),
\end{aligned}
$$

where

$$
\begin{aligned}
& S_{1}=\left\|u-\left(\sum_{i=0}^{m} u_{i}(t) t^{i}\right)\left(\sum_{j=0}^{n} v_{j}(t) t^{j}\right)\right\|, \\
& S_{2}=\left\|1-\left(\lambda-\sum_{i=0}^{m} u_{i}(t) t^{i}\right)\left(\sum_{j=0}^{n} v_{j}(t) t^{j}\right)\right\| .
\end{aligned}
$$

The term $\left\|u-\sum_{i=0}^{m} u_{i}(t) t^{i}\right\|$ can be bounded by $c_{m+1} t^{m+1}$ by assumption on the $u$. By construction of the $v_{j}$, the product $\left(\lambda-\sum_{i=0}^{m} u_{i}(t) t^{i}\right) \sum_{j=0}^{n} v_{j}(t) t^{j}$ is of the form

$$
1+(\text { terms of order } n+1 \text { in } t) \text {. }
$$

Hence, we find

$$
\left\|(\lambda-u(t))^{-1}-\sum_{j=0}^{n} v_{j}(t) t^{j}\right\|_{X} \leqslant C_{n+1}\left\|(\lambda-u(t))^{-1}\right\|_{X} t^{n+1}
$$

for some constant $C_{n+1}$. Since we assumed that $\lambda-u(t)$ was invertible for any $t$, inversion is a continuous map, and since $t$ belongs to a compact interval this norm can be bounded above.

Lemma 4.3 (action of bounded operators). Suppose that $u(t) \sim \sum_{i=0}^{\infty} u_{i}(t) t^{i}$ in $X_{\alpha}$ as $t \rightarrow 0^{+}$. Then an asymptotic expansion of $T_{j} u$ is given by

$$
T_{j} u(t) \sim \sum_{i=0}^{\infty} T_{j} u_{i}(t) t^{i} \quad \text { in } X
$$

as $t \rightarrow 0^{+}$. 
Proof. Since the $u_{j}$ are an asymptotic expansion of $u$ we have for every $m \in \mathbb{N}$ that

$$
\left\|u(t)-\sum_{i=0}^{m} u_{i}(t) t^{i}\right\|_{X_{\alpha}} \leqslant c_{m} t^{m+1}
$$

for some $c_{m} \in \mathbb{R}$. This means

$$
\left\|T_{j} u(t)-\sum_{i=0}^{m} T_{j} u_{i}(t) t^{i}\right\|_{X} \leqslant\left\|T_{j}\right\| c_{m} t^{m+1},
$$

and the claim follows from the boundedness of $T_{j}$ on $X_{\alpha}$.

\subsection{Holomorphic functional calculus}

Recall the holomorphic functional calculus in several variables.

Theorem 4.4 (Allan and Dales [2, theorem 9.3]). Let $X$ be a commutative unital Banach algebra, let $\boldsymbol{x}=\left(x_{1}, \ldots, x_{n}\right) \in X^{n}$ and let $U$ be an open neighbourhood of $\operatorname{Sp}(\boldsymbol{x})$ in $\mathbb{C}^{n}$. Then there is a continuous, unital homomorphism $\Theta_{\boldsymbol{x}}^{U}: O(U) \rightarrow X$ such that

(i) $\Theta_{\boldsymbol{x}}^{U}\left(Z_{j}\right)=x_{j}$ for $j=1, \ldots, n$ and

(ii) $\varphi\left(\Theta_{\boldsymbol{x}}^{U}(f)\right)=f\left(\varphi\left(x_{1}\right), \ldots, \varphi\left(x_{n}\right)\right)$

for every $f \in O(U)$ and every character $\varphi: X \rightarrow \mathbb{C}$. Here, $Z_{j}$ denotes the projection $Z_{j}(\boldsymbol{x})=x_{j}$.

The homomorphism $\Theta_{\boldsymbol{x}}^{U}$ is defined by Dunford integrals,

$$
\Theta_{\boldsymbol{x}}^{U}(f)=\left(\frac{1}{2 \pi \mathrm{i}}\right)^{n} \int_{\Gamma_{1}} \cdots \int_{\Gamma_{n}} f(z)\left(z_{1} 1-x_{1}\right)^{-1} \cdots\left(z_{n} 1-x_{n}\right)^{-1} \mathrm{~d} z_{1} \cdots \mathrm{d} z_{n},
$$

where $z=\left(z_{1}, \ldots, z_{n}\right) \in \mathbb{C}^{n}$, and $\Gamma_{i}$ is the circle $\left\{\left|z_{i}-w_{i}\right|=r_{i}\right\}$ with $r_{i}$ chosen so that $\operatorname{Sp}\left(x_{i}\right)$ is contained in the disc $B\left(w_{i}, r_{i}\right)$ for some $w_{i} \in \mathbb{C}$. This means that $\operatorname{Sp}(\boldsymbol{x})$ is contained in the open polydisc $B\left(w_{1}, r_{1}\right) \times \cdots \times B\left(w_{n}, r_{n}\right)$.

\subsection{Definition and properties of the forcing term}

We now make sense of the forcing term $F$ using holomorphic functional calculus. To this end, start with a function $\tilde{F} \in C([0, T]) \hat{\otimes}_{\pi} X \hat{\otimes}_{\pi} O(U)$ with $U$ as in hypothesis 3.6.

Define two linear evaluation maps: for $t \in[0, T]$ we set

$$
\begin{aligned}
E v_{t}: C([0, T]) & \rightarrow \mathbb{C}, \\
f & \mapsto f(t),
\end{aligned}
$$

and for $\boldsymbol{x}=\left(x_{0}, x_{1}, \ldots, x_{n}\right) \in X_{\alpha} \times X^{n}$ we set

$$
\begin{aligned}
E v_{\boldsymbol{x}}: O(U) & \rightarrow X, \\
f & \mapsto \Theta_{\left(x_{0}, x_{1}, \ldots, x_{n}\right)}^{U}(f),
\end{aligned}
$$

with functional calculus in $X$ using the fact that $X_{\alpha}$ is a subalgebra of $X$. 
To define the forcing term $F:[0, T] \times X_{\alpha} \times X^{n} \rightarrow X$ pointwise, choose $(t, \boldsymbol{x})$ with $t \in[0, T]$ and $\boldsymbol{x}=\left(x_{0}, x_{1}, \ldots, x_{n}\right) \in X_{\alpha} \times X^{n}$. Define a multilinear continuous map

$$
\begin{aligned}
\Phi_{(t, \boldsymbol{x})}: C([0, T]) \times X \times O(U) & \rightarrow X, \\
(f, y, g) & \mapsto E v_{t}(f) \cdot y \cdot E v_{\boldsymbol{x}}(g),
\end{aligned}
$$

with product in $X$. By the universal property of the projective tensor product (proposition 2.1) there is a well-defined linear map

$$
\bar{\Phi}_{(t, \boldsymbol{x})}: C([0, T]) \hat{\otimes}_{\pi} X \hat{\otimes}_{\pi} O(U) \rightarrow X .
$$

Now let $F$ act as

$$
\begin{aligned}
F:[0, T] \times X_{\alpha} \times X^{n} & \rightarrow X, \\
\left(t, x_{0}, x_{1}, \ldots, x_{n}\right) & \mapsto \bar{\Phi}_{(t, \boldsymbol{x})}(\tilde{F}) .
\end{aligned}
$$

The above definition ties in with the intuitive understanding of the action of $F$ on $X$. Recall from the properties of the projective tensor product that any $\tilde{F} \in C([0, T]) \hat{\otimes}_{\pi} X \hat{\otimes}_{\pi} O(U)$ can be represented as

$$
\tilde{F}=\sum_{j=0}^{\infty} \lambda_{j} f_{j} \otimes y_{j} \otimes g_{j}
$$

with $\lambda_{j} \in \mathbb{C}, f_{j} \in C([0, T]), y_{j} \in X$ and $g_{j} \in O(U)$. Moreover, $\sum_{j=1}^{\infty}\left|\lambda_{j}\right| \leqslant 1$ and $f_{j}, y_{j}, g_{j}$ tend to 0 as $j \rightarrow \infty$ in their respective spaces. Thus, by the above construction we have

$$
F\left(t, x, T_{1} x, \ldots, T_{n} x\right)=\sum_{j=0}^{\infty} \lambda_{j} f_{j}(t) y_{j} \Theta_{\left(x_{0}, T_{1} x, \ldots, T_{n} x\right)}^{U}\left(g_{j}\right)
$$

with products taken in $X$.

REMARK 4.5. A direct calculation shows that the map $F$ as just defined is continuous: if $x, x^{\prime} \in X_{\alpha}$ with $\|x\|_{X_{\alpha}},\left\|x^{\prime}\right\|_{X_{\alpha}} \leqslant R$, then there is a constant $c_{R}$ such that $\left\|F(t, x)-F\left(t, x^{\prime}\right)\right\|_{X} \leqslant c_{R}\left\|x-x^{\prime}\right\|_{X_{\alpha}}$ for all $t \in[0, T]$. This shows that the analyticity assumption in hypothesis 3.6(iii) implies the Lipschitz assumption of hypothesis $3.4($ iii)(a).

\subsection{Expansion of the Duhamel formula}

The integral equation (3.2) is the starting point for the construction of the asymptotic expansion of $u$. We now go through the following steps:

(1) expansion of $G(t) f$,

(2) expansion of the integral,

(3) recursive construction of the $u_{i}$. 
4.4.1. Expansion of $G(t) f$

By assumption (ii) of hypothesis 3.6 we can expand

$$
G(t) \sim G_{0}(t)+t G_{1}(t)+t^{2} G_{2}(t)+\cdots
$$

in $\mathcal{B}\left(X, X_{\alpha}\right)$. We then have the following.

Lemma 4.6. Define $f_{i}(t)=G_{i}(t) f$. Then $G(t) f \sim \sum_{i=0}^{\infty} f_{i}(t) t^{i}$ is an asymptotic expansion in $X_{\alpha}$ as $t \rightarrow 0^{+}$.

Proof. Fix $n \in \mathbb{N}$ and consider the difference of $G(t, 0) f$ and $\sum_{0}^{n} f_{i}(t) t^{i}$ in $X$ :

$$
\begin{aligned}
\left\|G(t) f-\sum_{i=0}^{n} f_{i}(t) t^{i}\right\|_{X_{\alpha}} & =\left\|G(t) f-\sum_{i=0}^{n} t^{i} G_{i}(t) f\right\|_{X_{\alpha}} \\
& =\left\|\left(G(t)-\sum_{i=0}^{n} t^{i} G_{i}(t)\right) f\right\|_{X_{\alpha}} \\
& \leqslant\left\|G(t)-\sum_{i=0}^{n} t^{i} G_{i}(t)\right\|_{\mathcal{B}\left(X, X_{\alpha}\right)}\|f\|_{X_{\alpha}} .
\end{aligned}
$$

As $G(t) \sim \sum_{i=0}^{\infty} G_{i}(t)^{i}$ in the operator norm, the result follows.

\subsubsection{Expansion of the integral}

Next we consider the integral in (3.2). We first construct an asymptotic expansion of $F\left(t, u(t), T_{1} u(t), \ldots, T_{n} u(t)\right)$ by a method similar to the symbol calculus of pseudo-differential operators. The idea is to find a formal inverse

$$
v \sim v_{0}(t)+v_{1}(t) t+v_{2}(t) t^{2}+\cdots
$$

of $\lambda-u$, i.e. $(\lambda-u) v=1$ in $X[t]$, and then use holomorphic functional calculus to define $F\left(t, u(t), T_{1} u(t), \ldots, T_{n} u(t)\right)$ by the Cauchy integral formula. Let $\lambda \notin \operatorname{Sp}\left(u_{0}\right)$, determine the formal product in $X_{\alpha}[t]$ in the relation

$$
\left(\lambda-\sum_{i=0}^{\infty} u_{i}(t) t^{i}\right) \sum_{i=0}^{\infty} v_{i}(t) t^{i}=1
$$

and collect terms according to order in $t$.

In our case, this yields the desired asymptotic expansion for $F$ as follows. Define the function $F\left(t, u(t), T_{1} u(t), \ldots, T_{n} u(t)\right)$ as

$$
\left(\frac{1}{2 \pi \mathrm{i}}\right)^{n+1} \int_{\Gamma} F(t, z)\left(z_{0} 1-u\right)^{-1}\left(z_{1} 1-T_{1} u\right)^{-1} \cdots\left(z_{n} 1-T_{n} u\right)^{-1} \mathrm{~d} z_{0} \cdots \mathrm{d} z_{n}
$$

for $\Gamma$ a suitable contour in $\mathbb{C}^{n+1}$ surrounding $\operatorname{Sp}(u(t)) \times \operatorname{Sp}\left(T_{1} u(t)\right) \times \cdots \times \operatorname{Sp}\left(T_{n} u(t)\right)$ and $\operatorname{Sp}\left(u_{0}(t)\right) \times \operatorname{Sp}\left(T_{1} u_{0}(t)\right) \times \cdots \times \operatorname{Sp}\left(T_{n} u_{0}(t)\right)$.

Setting $v^{(i)}(t)=\left(z_{i} 1-u^{(i)}(t)\right)^{-1}$ for $u^{(0)}=u$ and $u^{(i)}=T_{i} u$ with $i \in\{1, \ldots, n\}$, we construct an asymptotic expansion for $v^{(0)}(t) \cdots v^{(n)}(t)$ in the form $\sum b_{k}(t) t^{k}$. 
The proofs of lemmas 4.2 and 4.1 show that $v_{j}^{(i)}$ is of the form

$$
\sum_{k=0}^{j} \operatorname{polynomial}\left(u_{0}^{(i)}, \ldots, u_{j}^{(i)}\right)\left(z_{i} 1-u_{0}^{(i)}\right)^{-(k+1)},
$$

and hence the $b_{k}$ are products of such expansions. Thus, the definition

$$
F_{k}(t)=\left(\frac{1}{2 \pi \mathrm{i}}\right)^{n+1} \int_{\Gamma} F(t, z) b_{k}(t) \mathrm{d} z_{0} \cdots \mathrm{d} z_{n}
$$

makes sense as a Cauchy integral. Also, we have $v_{0}^{(i)}(t)=\left(z_{i} 1-u_{0}^{(i)}\right)^{-1}$, so

$$
F_{0}(t)=F\left(t, u_{0}, T_{1} u_{0}, \ldots, T_{n} u_{0}\right)
$$

in the lowest order.

We are now ready to iteratively construct an asymptotic expansion for $F$.

Lemma 4.7. Suppose that $m \in \mathbb{N}$ and there are constants $c_{m}^{(i)}$ and $i \in\{0, \ldots, n\}$ such that

$$
\left\|u(t)-\sum_{k_{0}=0}^{m} u_{k_{0}}^{(0)}(t) t^{k_{0}}\right\|_{X_{\alpha}} \leqslant c_{m}^{(0)} t^{m+1}
$$

and

$$
\left\|T_{i} u(t)-\sum_{k_{i}=0}^{m} u_{k_{i}}^{(i)}(t) t^{k_{i}}\right\|_{X} \leqslant c_{m}^{(i)} t^{m+1} .
$$

Then for $N \geqslant m$ we have

$$
\left\|F\left(t, u(t), T_{1} u(t), \ldots, T_{n} u(t)\right)-\sum_{k=0}^{N} F_{k}(t) t^{k}\right\|_{X} \leqslant c t^{m+1}
$$

for some constant $c>0$.

Proof. It follows that

$$
\begin{aligned}
\left\|F\left(t, u(t), T_{1} u(t), \ldots, T_{n} u(t)\right)-\sum_{k=0}^{N} F_{k}(t) t^{k}\right\|_{X} \\
=\|\left(\frac{1}{2 \pi \mathrm{i}}\right)^{n+1} \int_{\Gamma} F(t, z)\left(z_{0} 1-u(t)\right)^{-1} \cdots\left(z_{n} 1-T_{n} u(t)\right)^{-1} \mathrm{~d} z_{0} \cdots \mathrm{d} z_{n} \\
\quad-\left(\frac{1}{2 \pi \mathrm{i}}\right)^{n+1} \int_{\Gamma} F(t, z) \sum_{k=0}^{N} b_{k}(t) \mathrm{d} z_{0} \cdots \mathrm{d} z_{n} \|_{X} \\
=\left\|\left(\frac{1}{2 \pi \mathrm{i}}\right)^{n+1} \int_{\Gamma} F(t, z) R\left(u(t) ; z_{0}, \ldots, z_{n}\right) \mathrm{d} z_{0} \cdots \mathrm{d} z_{n}\right\|_{X} \\
\leqslant\left(\frac{1}{2 \pi \mathrm{i}}\right)^{n+1} \int_{\Gamma}|F(t, z)|\left\|R\left(u(t) ; z_{0}, \ldots, z_{n}\right)\right\|_{X} \mathrm{~d} z_{0} \cdots \mathrm{d} z_{n} \\
\leqslant d_{m} t^{m+1}\left(\frac{1}{2 \pi \mathrm{i}}\right)^{n+1} \int_{\Gamma}|F(t, z)| \mathrm{d} z_{0} \cdots \mathrm{d} z_{n}
\end{aligned}
$$


for a constant $d_{m}$ and

$$
R\left(u(t) ; z_{0}, \ldots, z_{n}\right)=\left(z_{0} 1-u(t)\right)^{-1} \cdots\left(z_{n} 1-T_{n} u(t)\right)^{-1}-\sum_{k=0}^{N} b_{k}(t) .
$$

As $\Gamma$ is compact and $F$ is continuous on $[0, T] \times \Gamma$, the result follows.

Now consider the integral

$$
\int_{0}^{t} G(s, t) F\left(s, u(s), T_{1} u(s), \ldots, T_{n} u(s)\right) \mathrm{d} s .
$$

We have seen that $G$ introduces additional terms into the asymptotic expansion. Formally, if $F(s)=\sum_{j=0}^{\infty} s^{j} F_{j}(s)$ and $G(t-s)=\sum_{j=0}^{\infty}(t-s)^{j} G_{j}(t-s)$, then

$$
G(t-s) F(s)=\sum_{j=0}^{\infty} \sum_{i=0}^{j}(t-s)^{i} s^{j-i} G_{i}(t-s) F_{j-i}(s),
$$

where we treat $t-s$ and $s$ as being the same order. Set

$$
\varphi_{j}(t, s)=\sum_{i=0}^{j}(t-s)^{i} s^{j-i} G_{i}(t-s) F_{j-i}(s)
$$

for $s \geqslant 0$. Then $\sum_{j=0}^{\infty} \varphi_{j}$ can be thought of as an asymptotic expansion of $G F$.

Lemma 4.8. Suppose that $m \in \mathbb{N}$ and there is a $c_{m}>0$ with

$$
\left\|u(t)-\sum_{i=0}^{m} u_{i}(z) z^{i}\right\|_{X_{\alpha}} \leqslant c_{m} t^{m+1} .
$$

Then for $N \geqslant m$ we have

$$
\left\|G(t-s) F(s)-\sum_{j=0}^{n} \varphi_{j}(t, s)\right\|_{X_{\alpha}} \leqslant c_{m} t^{m+1}
$$

for a constant $c_{m}>0$.

Note that through $F_{j}$, each $\varphi_{j}$ is a function

$$
\varphi_{j}=\varphi_{j}\left(t, s ; u_{0}, \ldots, u_{j}, T_{1} u_{0}, \ldots, T_{1} u_{j}, \ldots, T_{n} u_{0}, \ldots T_{n} u_{j}\right)
$$

of the $u_{0}, \ldots, u_{j}$ and the $T_{k} u_{0}, \ldots, T_{k} u_{j}$ for $k=1, \ldots, n$.

Proof. We have for $0 \leqslant s \leqslant t$ that

$$
\begin{gathered}
\left\|G(t-s) F-\sum_{j=0}^{N} \varphi_{j}\right\|_{X_{\alpha}} \\
=\|\left(G(t-s)-\sum_{j=0}^{N} G_{j}(t-s)(t-s)^{j}+\sum_{j=0}^{N} G_{j}(t-s)(t-s)^{j}\right) \\
\quad \times\left(F-\sum_{j=0}^{N} F_{j} s^{j}+\sum_{j=0}^{N} F_{j} s^{j}\right)-\sum_{j=0}^{N} \varphi_{j} \|_{X_{\alpha}}
\end{gathered}
$$


Short-time asymptotic expansions of semilinear evolution equations

$$
\begin{aligned}
& \leqslant\left\|\left(G(t-s)-\sum_{j=0}^{N} G_{j}(t-s)(t-s)^{j}\right)\left(F-\sum_{j=0}^{N} F_{j} s^{j}\right)\right\|_{X_{\alpha}} \\
& +\left\|\left(G(t-s)-\sum_{j=0}^{N} G_{j}(t-s)(t-s)^{j}\right) \sum_{j=0}^{N} F_{j} s^{j}\right\|_{X_{\alpha}} \\
& +\left\|\left(\sum_{j=0}^{N} G_{j}(t-s)(t-s)^{j}\right)\left(F-\sum_{j=0}^{N} F_{j} s^{j}\right)\right\|_{X_{\alpha}} \\
& +\left\|\left(\sum_{j=0}^{N} G_{j}(t-s)(t-s)^{j}\right)\left(\sum_{j=0}^{N} F_{j} s^{j}\right)-\sum_{j=0}^{N} \varphi_{j}\right\|_{X_{\alpha}} .
\end{aligned}
$$

By our previous results and by construction of the $\varphi_{j}$, each of the summands is bounded by $t^{m+1}$.

We note the following immediate corollary.

Corollary 4.9. Suppose that $m \in \mathbb{N}$ and there exists a $c_{m}>0$ with

$$
\left\|u(t)-\sum_{i=0}^{m} u_{i}(z) z^{i}\right\|_{X_{\alpha}} \leqslant c_{m} t^{m+1}
$$

Then, for $n \geqslant m$, we have

$$
\left\|\int_{0}^{t} G(t-s) F\left(s, u(s), T_{1} u(s), \ldots, T_{n} u(s)\right) \mathrm{d} s-\int_{0}^{t} \sum_{j=0}^{n} \varphi_{j} \mathrm{~d} s\right\|_{X_{\alpha}} \leqslant c_{m} t^{m+2}
$$

for a constant $c_{m}>0$.

So, formally, we have constructed an asymptotic expansion

$$
\begin{aligned}
\int_{0}^{t} G(t-s) & F\left(s, u(s), T_{1} u(s), \ldots, T_{n} u(s)\right) \mathrm{d} s \\
& \sim \sum_{j=0}^{\infty} \int_{0}^{t} \varphi_{j}\left(t, s ; u_{0}, \ldots, u_{j}, T_{1} u_{0}, \ldots, T_{1} u_{j}, \ldots, T_{n} u_{0}, \ldots, T_{n} u_{j}\right) \mathrm{d} s
\end{aligned}
$$

where each $u_{i}$ is a function of $s$.

\subsubsection{Recursive construction of the $u_{i}$}

To recursively construct the $u_{i}$ we expand both the left-hand and right-hand sides of (3.2) formally in powers of $z$ and match coefficients.

The left-hand side of (3.2) has the formal expansion

$$
u_{0}(t)+u_{1}(t) t^{2}+u_{2}(t) t^{2}+\cdots
$$


The right-hand side has the formal expansion

$$
\begin{aligned}
f_{0}(t)+ & f_{1}(t) t^{2}+f_{2}(t) t^{2}+\cdots \\
& +\int_{0}^{t} \varphi_{0}\left(t, s ; u_{0}, T_{1} u_{0}, \ldots, T_{n} u_{0}\right) \mathrm{d} s \\
& +t \int_{0}^{t} \varphi_{1}\left(t, s ; u_{0}, u_{1}, T_{1} u_{0}, \ldots, T_{n} u_{0}, T_{1} u_{1}, \ldots, T_{n} u_{1}\right) \mathrm{d} s \\
& +\cdots
\end{aligned}
$$

Now match the coefficients as follows:

$$
\begin{aligned}
& u_{0}(t)=f_{0}(t) \\
& u_{1}(t)=f_{1}(t)+\frac{1}{t} \int_{0}^{t} \varphi_{0}\left(t, s ; u_{0}, T_{1} u_{0}, \ldots, T_{n} u_{0}\right) \mathrm{d} s \\
& u_{2}(t)=f_{2}(t)+\frac{1}{t^{2}} \int_{0}^{t} \varphi_{1}\left(t, s ; u_{0}, u_{1}, T_{1} u_{0}, \ldots, T_{n} u_{0}, T_{1} u_{1}, \ldots, T_{n} u_{1}\right) \mathrm{d} s
\end{aligned}
$$

and so on. Here, $\varphi_{0}$ can be determined explicitly by (4.6) as

$$
\varphi_{0}=G_{0}(t-s) F\left(u_{0}(s), T_{1} u_{0}(s), \ldots, T_{n} u_{0}(s)\right),
$$

for example. Note that $u_{i}$ can be determined by knowing $u_{0}, \ldots, u_{i-1}$ and

$$
T_{1} u_{0}, \ldots, T_{1} u_{i-1}, \ldots, T_{n} u_{0}, \ldots, T_{n}, u_{i-1} .
$$

We must now show that this yields an asymptotic expansion (we omit the $u_{i}$ and $T_{j} u_{i}$ arguments of $\varphi$ to simplify the notation).

Proposition 4.10. Setting $u_{0}(t)=G_{0}(t) f$ and

$$
u_{i}(t)=f_{i}(t)+\frac{1}{t^{i}} \int_{0}^{t} \varphi_{i-1}(t, s) \mathrm{d} s
$$

for $i \geqslant 1$ yields an asymptotic expansion $u(t) \sim \sum_{i=0}^{\infty} u_{i} t^{i}$ in $X_{\alpha}$ as $t \rightarrow 0^{+}$.

Proof. We proceed by induction on $i$. For $u_{0}$ we have $\left\|u-u_{0}\right\|_{X_{\alpha}} \leqslant c t$ for some $c>0$ by estimating $G(t-s)-G_{0}(t-s)$.

Now suppose that for some $m>0$ we have

$$
\left\|u-\sum_{i=0}^{m} u_{i}(t) t^{i}\right\|_{X_{\alpha}} \leqslant c_{m} t^{m+1}
$$

for a $c_{m}>0$. By construction, we have

$$
u_{m+1}(t)=f_{m+1}(t)+\frac{1}{t^{m+1}} \int_{0}^{t} \varphi_{m}(t, s) \mathrm{d} s .
$$


Now, splitting each $u_{i}$ into parts corresponding to $f$ and to the integral term,

$$
\begin{aligned}
&\left\|u-\sum_{i=0}^{m+1} u_{i}(t) t^{i}\right\|_{X}=\left\|G(t) f+\int_{0}^{t} G(t-s) F(s, u(s)) \mathrm{d} s-\sum_{i=0}^{m+1} u_{i}(t) t^{i}\right\|_{X_{\alpha}} \\
&=\left\|G(t) f-\sum_{i=0}^{m+1} f_{i}(t) t^{i}\right\|_{X_{\alpha}} \\
& \quad+\left\|\int_{0}^{t} G(t-s) F(s, u(s)) \mathrm{d} s-\sum_{i=0}^{m} \int_{0}^{t} \varphi_{i}(t, s) \mathrm{d} s\right\|_{X_{\alpha}} \\
& \leqslant c_{1} t^{m+2}+c_{2} t^{m+2}
\end{aligned}
$$

for some constants $c_{1}$ and $c_{2}$ by lemma 4.6 and corollary 4.9 .

Proof of theorem 3.7. The claim is clear if $F$ is given by a finite sum

$$
F_{N}(t, x)=\sum_{j=0}^{N} \lambda_{j} f_{j}(t) y_{j} \Theta_{\left(x, T_{1} x, \ldots, T_{n} x\right)}^{U}\left(g_{j}\right) \text {. }
$$

Now suppose that $F$ is represented by an infinite sum

$$
F(t, x)=\sum_{j=0}^{\infty} \lambda_{j} f_{j}(t) y_{j} \Theta_{\left(x, T_{1} x, \ldots, T_{n} x\right)}^{U}\left(g_{j}\right) .
$$

The arguments also hold for the integrals by a convergence argument. The infinite sum converges in $X$ for every $(t, x) \in[0, T] \times X_{\alpha}$ and is continuous in $t$. Note that for fixed $x$ the Bochner integrals

$$
\int_{0}^{t} G(t-s) F_{N}(s, x) \mathrm{d} s
$$

converge in the norm to

$$
\int_{0}^{t} G(t-s) F(s, x) \mathrm{d} s \quad \text { for } F_{N} \rightarrow F
$$

in $C([0, T] ; X)$. This follows, for example, from the dominated convergence theorem for vector-valued integration (see [7, ch. III]), thus completing the proof.

\section{Examples}

We illustrate two applications of the framework: first to Dirichlet problems on bounded domains and then to Markovian BSDEs. The examples highlight that our approach can yield an approximation of both the function and its gradient.

\subsection{Semilinear evolution equations on bounded domains}

We discuss an economically motivated example in the context of pricing bonds depending on climate risk [17]. The market price of climate risk is expressed as the derivative of a PDE solution and we wish to give an approximation of that price. In two dimensions we consider a forcing term quadratic in the gradient, i.e. the function $F\left(t, T_{1} u, T_{2} u\right)$ is quadratic in the $T_{j} u$. 


\subsubsection{Set-up}

Let $\Omega$ be a bounded domain in $\mathbb{R}^{2}$ with smooth boundary $\partial \Omega$, and consider the semilinear Dirichlet problem

$$
\left.\begin{array}{rl}
\partial_{t} u=A u+F\left(t, T_{1} u, T_{2} u\right) \quad \text { on }(0, T] \times \Omega, \\
u=0 \quad \text { on }(0, T] \times \partial \Omega, \\
u=f \quad \text { on }\{0\} \times \Omega,
\end{array}\right\}
$$

for the differential operators

$$
\begin{gathered}
A=\left(\sigma_{1}(x)^{2} \partial_{1}^{2}+\sigma_{2}(x)^{2} \partial_{2}^{2}\right)+\left(\mu_{1}(x) \partial_{1}+\mu_{2}(x) \partial_{2}\right), \\
T_{1}=\sigma_{1}(x) \partial_{1}, \quad T_{2}=\sigma_{2}(x) \partial_{2},
\end{gathered}
$$

where the $\sigma_{i}$ and $\mu_{i}$ are bounded smooth functions $\bar{\Omega} \rightarrow \mathbb{R}$. For simplicity we assume that $A$ is uniformly elliptic in the sense that

$$
\sigma_{1}(x)^{2} \xi_{1}^{2}+\sigma_{2}(x)^{2} \xi_{2}^{2} \geqslant c\left(\xi_{1}^{2}+\xi_{2}^{2}\right)
$$

for some $c$ and all $x \in \Omega, \xi \in \mathbb{R}^{2}$.

This implies that $A$ is sectorial and extends to the generator of an analytic semigroup $G(t)$ in the Banach space $X=C(\bar{\Omega})$ (see [20, ch. 3.1.5]). Set $\alpha=\frac{1}{2}$ and $X_{\alpha}=C_{\mathcal{B}}^{1}(\bar{\Omega})$ leading to a continuous inclusion $X_{\alpha} \hookrightarrow X$. Pick the initial datum $f$ in $C_{\mathcal{B}}^{1}(\bar{\Omega})=\left\{u \in C^{1}(\bar{\Omega}):\left.u\right|_{\partial \Omega}=0\right\}$.

Set

$$
\theta(t, x)=\frac{\mu_{1}(t, x)}{\sigma_{1}(t, x)}
$$

expressing the market price of financial risk. Clearly, $\theta \in C([0, T]) \hat{\otimes}_{\pi} C(\bar{\Omega})$. Choose $\varphi \in C([0, T]) \hat{\otimes}_{\pi} C(\bar{\Omega})$, representing a yield curve used for discounting cashflows. Define the forcing term by

$$
F\left(t, x, z_{1}, z_{2}\right)=-\theta(t, x) z_{1}^{2}-\frac{1}{2} z_{2}^{2}-\varphi(t, x)-\frac{1}{2} \theta(t, x)^{2},
$$

so that $F$ satisfies the continuity condition in hypothesis 3.4(iii)(a) and is holomorphic on $\mathbb{C}^{n}$, satisfying hypothesis 3.6(iii).

Objective. The aim is now to find an asymptotic expansion not of $u$ but of $T_{1} u$ : this is the quantity that represents the market price of climate risk. Moreover, we wish to be able to see explicitly how the data $\theta, \varphi$ enter the solution.

Algebra. It is clear that $X$ is a unital Banach algebra under the supremum norm, with the unit given by the function, which is identical to 1 everywhere.

Geometry. It remains to find an expansion of $G(t)$ in $\mathcal{B}\left(X, X_{\alpha}\right)$. The tricky part is that the manifold $\bar{\Omega}$ is a compact manifold with boundary. We can view the set $\Omega$ as being an open domain in a compact manifold $M$ without boundary, such as the 2-torus $\mathbb{R}^{2} / \mathbb{Z}^{2}$. Without loss of generality, $\Omega$ lies within a single coordinate patch. Extend the operator $A$ to the whole of $M$ such that outside a neighbourhood of $\Omega$ the operator becomes the standard Laplacian on $M$ (see $[21, \S 3]$ ). 
By [13, theorem 2.4.2], the heat kernel of $G(t)$ can be expressed as $h(t, x, y)=$ $h_{\Omega}(t, x, y)-h_{\partial \Omega}(t, x, y)$, where $h_{\Omega}$ expresses the heat kernel on $M$ in the coordinate patch containing $\Omega$, and $h_{\partial \Omega}$ is the compensating term reflecting the boundary conditions. Both kernels are smooth and decay rapidly in the space variables.

By $\left[4\right.$, theorem 2.30], there are smooth functions $\Phi_{j}(x, y)$ such that

$$
h_{\Omega}(t, x, y) \sim q_{t}(x, y)\left(\Phi_{0}(x, y)+\Phi_{1}(x, y) t+\Phi_{2}(x, y) t^{2}+\cdots\right)
$$

with

$$
q_{t}(x, y)=\frac{1}{4 \pi t} \exp \left(-\frac{d(x, y)^{2}}{4 t}\right)
$$

for the geodesic distance $d(x, y)$ under the Riemannian metric $g$ induced by $A$. The geodesic distance $d$ can be made explicit after a change of variables: the inverse metric tensor $g^{i j}(x)$ is given by the matrix

$$
\left(\begin{array}{cc}
\sigma_{1}(x)^{2} & 0 \\
0 & \sigma_{2}(x)^{2}
\end{array}\right)
$$

Thus, setting

$$
x_{1}^{\prime}=\int^{x_{1}} \sigma_{1}(\eta)^{2} \mathrm{~d} \eta \text { and } x_{2}^{\prime}=\int^{x_{2}} \sigma_{2}(\eta)^{2} \mathrm{~d} \eta
$$

yields $q_{t}\left(x^{\prime}, y^{\prime}\right)=(4 \pi t)^{-1} \exp \left(-\left\|x^{\prime}-y^{\prime}\right\| / 4 t\right)$, with the usual Euclidean distance $\|\cdot\|$ in $\mathbb{R}^{2}$.

The series (5.2) is asymptotic in the sense of definition 3.1 in the Banach space $C^{k}(\bar{\Omega})$ for every $k \geqslant 0$. Let $G_{0}$ be the operator with kernel $q_{t}(x, y) \Phi_{0}(x, y)-h_{\partial \Omega}$ and let $G_{j}(t)$ be the operator on $X$ with integral kernel $q_{t}(x, y) \Phi_{j}(x, y)$.

It is easy to see from the smoothness of the $q_{t}(x, y) \Phi_{j}(x, y)$ and $h_{\partial \Omega}$ that the $G_{j}$ belong to the bounded operators $\mathcal{B}\left(X, X_{\alpha}\right)$.

Asymptotics. Applying theorem 3.7, we obtain, in the lowest orders,

$$
\begin{aligned}
& u_{0}(t)=G_{0}(t) f \\
& u_{1}(t)=G_{1}(t) f+\frac{1}{t} \int_{0}^{t} G_{0} F_{0}(s) \mathrm{d} s \\
& u_{2}(t)=G_{2}(t) f+\frac{1}{t^{2}} \int_{0}^{t} s G_{0}(t-s) F_{1}(s)+(t-s) G_{1}(t-s) F_{0}(s) \mathrm{d} s,
\end{aligned}
$$

where

$$
\begin{aligned}
& F_{0}(s)=-\theta(s)\left(T_{1} u_{0}(s)\right)^{2}-\frac{1}{2}\left(T_{2} u_{0}(s)\right)^{2}-\varphi(s)-\frac{1}{2} \theta(s)^{2} \\
& F_{1}(s)=-2 \theta(s) T_{1} u_{0}(s) T_{1} u_{1}(s)-T_{2} u_{0}(s) T_{2} u_{1}(s)
\end{aligned}
$$

by direct calculation. Hence, $T_{1} u \sim T_{1} u_{0}+t T_{1} u_{1}+\cdots$, with controllable convergence in $X$ yielding the desired approximation of the market price of climate risk. 
Discussion. It is clear that the above expansion may not be the most suitable version for numerical studies. However, it has several benefits that make it useful from a qualitative point of view. First, the expansion is tractable and can easily be performed to arbitrary order. Second, it yields an explicit insight into the effect of the data $\theta$ and $\varphi$. This is useful for assessing the growth of the function $T_{1} u$ and the sensitivities with respect to changes in the data. Third, and most importantly, it can be used calibrate the model to market data, i.e. to infer the behaviour of $\theta$ and $\varphi$ from observed market data for small times $t$.

In practical applications, the kernel $h_{\partial \Omega}$ is often neglected in applications, as it decreases exponentially with distance from the boundary (the 'principle of not feeling the boundary'; see [18]).

Also, elegant as the construction of the heat kernel (5.2) may be, concrete computations are difficult, since the terms depend on the geodesic distance. Instead, one can apply pseudo-differential methods to obtain an explicit heat kernel expansion as in [28, ch. 7.13] or in the Boutet de Monvel calculus [15].

REMARK 5.1. One can extend the above considerations to Cauchy problems defined on $\mathbb{R}^{n}$. Let $\Omega \subset \mathbb{R}^{n}$ be bounded and open and denote by $P$ the corresponding projection $C^{k}\left(\mathbb{R}^{n}\right) \rightarrow C^{k}(\bar{\Omega})$. Let $h(t, \xi, \eta) \sim \sum_{j=0}^{\infty} h_{j}(t, \xi, \eta)$ be the heat kernel expansion on $\mathbb{R}^{n}$ valid locally uniformly and define by $G, G_{j}$ linear operators acting on $C^{k}\left(\mathbb{R}^{n}\right)$ with integral kernels $h$ and $h_{j}$, respectively. Then $P G P \sim \sum_{j=0}^{\infty} P G_{j} P$ in the corner algebra $P \mathcal{B}\left(X, X_{\alpha}\right) P$. Hence, $P u \sim P u_{0}+t P u_{1}+t^{2} P u_{2}+\cdots$, with $u_{j}$ computed as before, with $G, G_{j}$ replaced by $P G P$ and $P G_{j} P$, respectively. Again, if the PDE solution is considered at points away from the boundary, one can often ignore the boundary effect altogether.

\subsection{Connection with backward stochastic differential equations}

We indicate how our framework can be applied to BSDEs. These were introduced in the linear case in [5] and the theory has found applications in stochastic optimal control, PDEs [23], financial mathematics [9] and nonlinear expectations [8]. We refer the reader to [24] for detailed references.

Loosely speaking, a solution of a BSDE is a pair of adapted stochastic processes $(Y, Z)$ with the terminal condition $Y_{T}=\xi$ such that

$$
-\mathrm{d} Y_{t}=F\left(t, Y_{t}, Z_{t}\right) \mathrm{d} t-Z_{t}^{\mathrm{T}} \mathrm{d} W_{t}, \quad Y_{T}=\xi
$$

or, equivalently,

$$
Y_{t}=\xi+\int_{t}^{T} F\left(r, Y_{r}, Z_{r}\right) \mathrm{d} r-\int_{t}^{T} Z_{r}^{\mathrm{T}} \mathrm{d} W_{r},
$$

where $W_{t}$ denotes a multidimensional Brownian motion. The function $F$ is called the driver of the BSDE.

We are interested in the short-time behaviour of the pair $(Y, Z)$. The literature on asymptotic expansions in the case of linear SDEs is much richer than that in the BSDE context. One can broadly identify two strands: those using probabilistic methods (the Itō-Watanabe formula as an analogue to Taylor's theorem), and those based on functional analytic perturbation methods. For the former we refer to [22] 
for comprehensive references in the SDE case, with a recent extension to BSDEs in [11]. Perturbation methods based on Malliavin calculus were used in a BSDE context in [27], where the lowest order terms of an asymptotic expansion of a BSDE solution are described as BSDE solutions themselves. To our knowledge, heat kernel methods (which have found application in finance mostly in relation to implied volatility $[10,12,16])$ seem not to have been applied to nonlinear BSDEs.

We specialize to the case where the driver depends on the underlying probability space through a diffusion process $X$ and the terminal condition is a function of $X$. The BSDE is called Markovian in this case. It is expressed as a forward-backward system of a stochastic differential equation (SDE) and a BSDE, and leads to a PDE.

To this end we make the following assumptions on the coefficients and data (see $[9, \S 4])$. Let $(\Omega, \mathcal{F}, \mathbb{P})$ be the standard filtered probability space of $n$-dimensional Brownian motion. Denote by $\mathcal{F}_{r}^{t}$ be the completion of the $\sigma$-algebra $\sigma\left\{W_{s}-W_{t}: t \leqslant\right.$ $s \leqslant r\}$ with the $\mathbb{P}$-null sets of $\mathcal{F}$.

HYPOTHESIS 5.2.

(i) Domain and image: we have

$$
\begin{aligned}
& b:[0, T] \times \mathbb{R}^{p} \rightarrow \mathbb{R}^{p}, \\
& \sigma:[0, T] \times \mathbb{R}^{p} \rightarrow \mathbb{R}^{p \times n}, \\
& F:[0, T] \times \mathbb{R}^{p} \times \mathbb{R} \times \mathbb{R}^{n} \rightarrow \mathbb{R}, \\
& f: \mathbb{R}^{p} \rightarrow \mathbb{R},
\end{aligned}
$$

and all these maps are assumed to be measurable.

(ii) Growth: there is a constant $c>0$ such that

$$
\begin{aligned}
|b(t, x)|+|\sigma(t, x)| & \leqslant c(1+|x|), \\
|F(t, x, y, z)|+|f(x)| & \leqslant c\left(1+|x|^{r}\right),
\end{aligned}
$$

for some $r \geqslant \frac{1}{2}$ and all $(t, x, y, z) \in[0, T] \times \mathbb{R}^{p} \times \mathbb{R} \times \mathbb{R}^{n}$.

(iii) Continuity: there is a constant $c>0$ such that

$$
\begin{gathered}
|\sigma(t, x)-\sigma(t, y)|+|b(t, x)-b(t, y)| \leqslant c(1+|x-y|), \\
\left|F\left(t, x, y_{1}, z_{1}\right)-F\left(t, x, y_{2}, z_{2}\right)\right| \leqslant c\left(\left|y_{1}-y_{2}\right|+\left|z_{1}-z_{2}\right|\right) \\
\text { for }\left(t, x, y_{i}, z_{i}\right) \in[0, T] \times \mathbb{R}^{p} \times \mathbb{R} \times \mathbb{R}^{n} \text { and } i
\end{gathered}
$$

The first ingredient of a forward-backward system is the SDE

$$
\left.\begin{array}{rlrl}
\mathrm{d} X_{t} & =b\left(t, X_{t}\right) \mathrm{d} t+\sigma\left(t, X_{t}\right) \mathrm{d} W_{t}, & & s \leqslant t \leqslant T, \\
X_{t} & =x, & & 0 \leqslant t \leqslant s,
\end{array}\right\}
$$

on $(t, x) \in[0, T] \times \mathbb{R}^{p}$, where $T$ is the final time. We denote the unique strong solution of (5.3) by $X_{t}^{s, x}$ for $0 \leqslant t \leqslant T$ (see [26, definition 10.9]). We can assume that $X_{t}^{s, x}$ is almost surely jointly continuous in the variables $(s, t, x)$. The second ingredient is the BSDE

$$
\left.\begin{array}{rl}
-\mathrm{d} Y_{t} & =F\left(t, X_{t}, Y_{t}, Z_{t}\right) \mathrm{d} t-Z_{t}^{\mathrm{T}} \mathrm{d} W_{t}, \\
Y_{T} & =f\left(X_{T}\right),
\end{array}\right\}
$$


for $t \in[0, T]$. Here $Z_{t}^{\mathrm{T}}$ denotes the transpose of the vector-valued process $Z_{t}$ taking values in $\mathbb{R}^{n}$. For simplicity we restrict ourselves to scalar-valued $Y_{t}$ although the results can be generalized to vector-valued processes.

A solution of the forward-backward system (5.3) and (5.4) is given by a triple $\left(X_{t}^{s, x}, Y_{t}^{s, x}, Z_{t}^{s, x}\right)$, with $\left(Y_{t}^{s, x}, Z_{t}^{s, x}\right)$ a solution of (5.4) or, equivalently, of the integral equation

$$
Y_{t}=f\left(X_{T}\right)+\int_{t}^{T} F\left(r, X_{r}, Y_{r}, Z_{r}\right) \mathrm{d} r-\int_{t}^{T} Z_{r}^{\mathrm{T}} \mathrm{d} W_{r}
$$

We require that $Y_{t}$ be a continuous adapted process, and $Z_{t}$ be a predictable process with

$$
\int_{0}^{T}\left|Z_{r}\right|^{2} \mathrm{~d} s<\infty
$$

almost surely. The process $Y_{t}^{s, x}$ has a version that is almost surely continuous in $(s, t)$ and twice continuously differentiable in $x$. For technical details we refer the reader to [23].

The link with semilinear PDEs is a theorem of Feynman-Kac type.

TheOrem 5.3 (El Karoui and Mazliak [8, proposition 3.5]). Let u be a function belonging to $C^{1,2}\left([0, T] \times \mathbb{R}^{p}\right)$ and suppose there is a constant $c$ such that

$$
|u(t, x)|+\left|\sigma(t, x)^{\mathrm{T}} D u(t, x)\right| \leqslant c(1+|x|)
$$

for $(t, x) \in[0, T] \times \mathbb{R}^{p}$. Also suppose that $u$ is a solution of the PDE

$$
\left.\begin{array}{rl}
\partial_{t} u+\mathcal{A}(t) u+F\left(t, x, u, \sigma^{\mathrm{T}} D u\right) & =0, \\
u(T, x) & =f(x),
\end{array}\right\}
$$

for the differential operator

$$
\mathcal{A}(t)=\frac{1}{2} \sum_{i, j} a_{i j}(t, x) \partial_{i} \partial_{j}+\sum_{i} b_{i}(t, x) \partial_{i}
$$

where $a_{i j}=\left[\sigma \sigma^{\mathrm{T}}\right]_{i j}$ and $D$ is the gradient operator $D=\left(D_{1}, \ldots, D_{p}\right)$. Then $u(t, x)=Y_{t}^{(t, x)}$, where $\left\{\left(Y_{t}^{(s, x)}, Z_{t}^{(s, x)}\right): s \leqslant t \leqslant T\right\}$ is the unique solution of the $B S D E$ (5.4). Moreover,

$$
\left.\begin{array}{rl}
Y_{t}^{s, x} & =u\left(t, X_{t}^{s, x}\right) \\
Z_{t}^{s, x} & =\sigma\left(t, X_{t}^{s, x}\right)^{\mathrm{T}} D u\left(t, X_{t}^{s, x}\right)
\end{array}\right\}
$$

for $s \leqslant t \leqslant T$.

The boundedness condition (5.5) will be irrelevant in our context, as we shall work in uniform function spaces or in Sobolev spaces on bounded domains. Related results can be formulated for bounded domains with boundary conditions.

After a time reversion $\tau=T-t$ the PDE (5.6) fits into our framework with

$$
T_{j}=\sigma_{1 j} D_{1}+\cdots+\sigma_{p j} D_{p}
$$


for $j=1, \ldots, n$ viewing the matrix components $\sigma_{i j}$ as multiplication operators. We let $X=C\left(\mathbb{R}^{p}\right)$ and $X_{\alpha}=C^{1}\left(\mathbb{R}^{p}\right)$. We assume that $\mathcal{A}$ is independent of time and sectorial in $X$ so it generates an analytic semigroup $G(t)$ in $X$ (see [20, ch. 3.1.2]). For ease of exposition we consider the simple perturbation approach on $\mathbb{R}^{n}$, where $G_{0}=G, G_{j}=0$ for $j \geqslant 1$. This satisfies hypothesis 3.6(ii).

THEOREM 5.4. Suppose hypotheses 3.4 and 3.6 are met. Assume that the derivatives of $F\left(t, x, z_{0}, z_{1}, \ldots, z_{n}\right)$ with respect to $x$ of all orders are bounded uniformly in $z_{1}, \ldots, z_{n}$. Then there are processes $Y_{t}^{(i)}$ with values in $\mathbb{R}$ and $Z_{t}^{(i)}$ with values in $\mathbb{R}^{n}$ such that for every $m \in \mathbb{N}$ there is a constant $c_{m} \geqslant 0$ with

$$
\left|Y_{t}^{s, x}-\sum_{i=0}^{m} Y_{t}^{(i)}\right| \leqslant c_{m}(t-s)^{m+1}, \quad\left|Z_{t}^{s, x}-\sum_{i=0}^{m} Z_{t}^{(i)}\right| \leqslant c_{m}(t-s)^{m+1}
$$

almost surely for all sufficiently small t. The processes $Y_{t}^{(i)}$ are given explicitly as

$$
\begin{aligned}
Y_{t}^{(0)} & =\mathbb{E}\left(f\left(X_{T}^{s, x}\right)\right), \\
Y_{t}^{(i)} & =\mathbb{E}\left(\int_{t}^{T} \bar{F}_{i-1}\left(r ; X_{t}^{s, x}, Y_{t}^{0}, Z_{t}^{0}, \ldots, Y_{t}^{i-1}, Z_{t}^{i-1}\right) \mathrm{d} r\right),
\end{aligned}
$$

for $i \geqslant 1$ and functions $\bar{F}_{i}$ that can be constructed from the $F_{i}$ in theorem 3.7 .

Proof. We proceed in two steps, and for ease of exposition set $s=0$.

STEP 1 (existence of the approximation). By theorem 3.7 there exist functions $u_{i}$ : $[0, T] \rightarrow C^{1}\left(\mathbb{R}^{p}\right)$ such that

$$
\begin{aligned}
u(t) & \sim u_{0}(t)+u_{1}(t) t+u_{2}(t) t^{2}+\cdots, \\
T_{j} u(t) & \sim T_{j} u_{0}(t)+T_{j} u_{1}(t) t+T_{j} u_{2}(t) t^{2}+\cdots,
\end{aligned}
$$

for $j=1, \ldots, n$ in the spaces $C\left(\mathbb{R}^{p}\right)$ and $C^{1}\left(\mathbb{R}^{p}\right)$, respectively. Then set

$$
\begin{aligned}
Y_{t}^{(i)} & =t^{i} u_{i}\left(t, X_{t}^{0, x}\right), \\
Z_{t}^{(i)} & =t^{i}\left(T_{1} u_{i}\left(t, X_{t}^{0, x}\right), \ldots, T_{n} u_{i}\left(t, X_{t}^{0, x}\right)\right)^{\mathrm{T}} .
\end{aligned}
$$

Since the approximation of $u$ by $\sum_{i=0}^{\infty} u_{i}(t) t^{i}$ is formulated in the uniform norm, the claim on the almost sure approximation follows similarly for $T_{j} u$.

STEP 2 (representation as a BSDE solution [23, p. 202]). Every $t^{i} u_{i}(t)$ is a mild solution of the PDE (3.5). By assumption, all derivatives of $F$ are bounded so that the forcing term $F_{i}$ is also bounded. Hence, the PDE (3.5) has a unique classical solution in $C^{1,2}\left([0, T] \times \mathbb{R}^{p}\right)$ and this must agree with the mild solution. But then by the Feynman-Kac theorem (theorem 5.3) we have that for $i \geqslant 1$ the process $Y_{t}^{(i)}=t^{i} u_{i}\left(t, X_{t}^{0, x}\right)$ is the unique solution of the BSDE

$$
\begin{aligned}
-\mathrm{d} Y_{t}^{(i)}=F_{i-1}\left(t ; X_{t}^{0, x},\right. & u_{0}, \ldots, u_{i-1} \\
& \left.T_{1} u_{0}, \ldots, T_{1} u_{i-1}, \ldots, T_{n} u_{0}, \ldots, T_{n} u_{i-1}\right) \mathrm{d} t-Z_{t}^{(i) \mathrm{T}} \mathrm{d} W_{t},
\end{aligned}
$$


where each $T_{k} u_{i}$ is evaluated at $\left(t, X_{t}^{0, x}\right)$. We can shorten this to

$$
\left.\begin{array}{rl}
-\mathrm{d} Y_{t}^{(i)} & =\bar{F}_{i-1}\left(t ; X_{t}^{t, x}, Y_{t}^{0}, Z_{t}^{0}, \ldots, Y_{t}^{i-1}, Z_{t}^{i-1}\right) \mathrm{d} t-Z_{t}^{(i) \mathrm{T}} \mathrm{d} W_{t}, \\
Y_{T} & =0
\end{array}\right\}
$$

for $\bar{F}_{i-1}$ obtained from $F_{i-1}$ by scaling the arguments by negative powers of $t$. The solution of this BSDE is given explicitly as in (5.9). The process $Z_{t}^{(i)}$ is the unique process satisfying

$$
\int_{t}^{T} Z_{r}^{(i)} \mathrm{d} W_{r}=\int_{t}^{T} \bar{F}_{i-1} \mathrm{~d} r-\mathbb{E}\left(\int_{t}^{T} \bar{F}_{i-1} \mathrm{~d} r\right)
$$

which follows form the representation theorem for Brownian martingales [26, theorem 36.1]. (There is also an alternative representation of $Z^{(i)}$ as an expectation which is more suitable for numerical simulations; see [3].) The process $Y_{t}^{(0)}$ is given by $[G(t) f]\left(X_{t}^{0, x}\right)$, which can be expressed as the expectation (5.8).

REMARK 5.5. The scheme serves to analytically approximate the solution of a forward-backward system so as to allow qualitative insights into the structure of the solution. A numerical implementation of this scheme can be done with wellestablished and efficient methods for numerically simulating BSDEs (see, for example, [25]). Also, some practical problems do not involve sufficiently regular terminal conditions of drivers. By standard stability results of BSDE theory (e.g. [24, theorem 5.11]), one can approximate the original problem by one involving continuous and analytic functions and then employ our asymptotic expansion scheme.

\section{References}

1 R. A. Adams and J. J. F. Fournier. Sobolev spaces, 2nd edn (Oxford: Academic Press, 2003).

2 G. R. Allan and H. G. Dales. Introduction to Banach spaces and algebras (Oxford University Press, 2011).

3 J. Ma. and J. Zhang. Representation theorems for backward stochastic differential equations. Ann. Appl. Prob. 12 (2002), 1390-1418.

4 N. Berline, E. Getzler and M. Vergne. Heat kernels and Dirac operators (Springer, 1992).

5 J.-M. Bismut. Conjugate convex functions in optimal stochastic control. J. Math. Analysis Applic. 44 (1973), 384-404.

6 O. Calin, D. Chang, K. Furutani and C. Iwasaki. Heat kernels for elliptic and sub-elliptic operators (New York: Birkhäuser, 2011).

$7 \quad$ N. Dunford and J. T. Schwartz. Linear operators, Vol. 1: General theory (Wiley, 1957).

$8 \quad$ N. El Karoui and L. Mazliak. Backward stochastic differential equations, Pitman Research Notes in Mathematics, vol. 364 (Harlow: Longman, 1997).

9 N. El Karoui, S. Peng and M. C. Quenez. Backward stochastic differential equations in finance. Math. Fin. 7 (1997), 1-71.

10 M. Forde and A. Jacquier. Small-time asymptotics for implied volatility under the Heston model. Int. J. Theor. Appl. Finan. 12 (2009), 861-876.

11 M. Fujii and A. Takahashi. Analytical approximation for non-linear FBSDEs with perturbation scheme. Int. J. Theor. Appl. Finan. 15 (2012), 1250034.

12 J. Gatheral, E. P. Hsu, P. Laurence, C. Ouyang and T. H. Wang. Asymptotics of implied volatility in local volatility models. Math. Fin. 22 (2012), 591-620.

13 G. Greiner. An asymptotic expansion for the heat equation. Arch. Ration. Mech. Analysis 41 (1971), 163-218. 
14 A. Grothendieck. Produits tensoriels topologiques et espaces nucléaires. Memoirs of the American Mathematical Society, vol. 16 (Providence, RI: American Mathematical Society, 1955).

15 G. Grubb. Functional calculus of pseudodifferential boundary problems, 2nd edn (Boston, MA: Birkhäuser, 1996).

16 P. Henry-Labordère. Analysis, geometry, and modeling in finance: advanced methods in option pricing (Boca Raton, FL: CRC Press, 2008).

17 U. Horst and M. Müller. On the spanning property of risk bonds priced by equilibrium. Math. Operat. Res. 32 (2007), 784-807.

18 E. P. Hsu. On the principle of not feeling the boundary for diffusion processes. J. Lond. Math. Soc. 51 (1995), 373-382.

19 H. Kumano-Go. Pseudo-differential operators (Cambridge, MA: MIT Press, 1981).

20 A. Lunardi. Analytic semigroups and optimal regularity in parabolic problems (Basel: Birkhäuser, 1995).

21 H. P. McKean and I. M. Singer. Curvature and the eigenvalues of the Laplacian. J. Diff. Geom. 1 (1967), 43-69.

22 P. Malliavin and A. Thalmaier. Stochastic calculus of variations in mathematical finance (Springer, 2006).

23 E. Pardoux and S. Peng. Backward stochastic differential equations and quasilinear parabolic partial differential equations. Lecture Notes in Control and Information Science, vol. 176, pp. 200-217 (Springer, 1992).

24 E. Pardoux and A. Răşcanu. Stochastic differential equations, backward SDEs, partial differential equations (Springer, 2014).

25 A. Richou. Numerical simulation of BSDEs with drivers of quadratic growth. Annals Appl. Prob. 21 (2011), 1933-1964.

26 L. C. G. Rogers and D. Williams. Diffusions, Markov processes and martingales, vol. 2: Itō calculus (Cambridge University Press, 2000).

27 A. Takahashi and T. Yamada. A remark on approximation of the solutions to partial differential equations in finance. In Recent advances in financial engineering 2011: Proc. Int. Workshop on Finance 2011 (ed. A. Takahashi, Y. Muromachi and H. Nakaoka), pp. 133181 (Singapore: World Scientific, 2012).

28 M. E. Taylor. Partial differential equations, II: Qualitative solutions of linear equations, 2nd edn (Springer, 2011)

29 M. E. Taylor. Partial differential equations, III: Nonlinear equations, 2nd edn (Springer, 2011)

30 D. V. Vassilevich. Heat kernel expansion: user's manual. Phys. Rep. 388 (2003), 279-360. 